\title{
« Dans le malheur on ne peut pas être seul avec Dieu ». De l'origine et de la nature des tinni goin (Burkina Faso)
}

"In Dire Straits, One Cannot Be Alone with God »: About the Origin and Nature of the Guin Tinni (Burkina Faso) »

\section{Michèle Dacher}

\section{OpenEdition}

Journals

Édition électronique

URL : http://journals.openedition.org/span/1019

DOI : 10.4000/span.1019

ISSN : 2268-1558

Éditeur

École pratique des hautes études. Sciences humaines

Édition imprimée

Date de publication : 1 septembre 1987

Pagination : 69-110

ISSN : 0294-7080

Référence électronique

Michèle Dacher, « «Dans le malheur on ne peut pas être seul avec Dieu ». De l'origine et de la nature des tinni goin (Burkina Faso) », Systèmes de pensée en Afrique noire [En ligne], 8| 1987, mis en ligne le 16 octobre 2013, consulté le 19 avril 2019. URL : http://journals.openedition.org/span/1019 ; DOI : 10.4000/span. 1019 


\section{"DANS IE MALHEUR ON NE PEUT PAS ETRE SEUL AVEC DIEU" \\ DE L'ORIGINE ET DE LA NATURE DES TINNI GOIN (BURKINA FASO) ${ }^{1}$}

par

Michèle Dacher

INTRODUCTION

Les Goin qui parlent français ${ }^{2}$ nomment "fétiche" tout ce sur quoi ils sacrifient, tout ce qui peut être support d'une puissance extra-humaine. Or il suffit de confier un voeu à n'importe quel objet naturel ou fabriqué pour qu'il se charge, à des degrés divers, de cette puissance. L'univers entier peut donc être "fétichisé".

Deux termes goin permettent de cerner cette notion de puissance: fango, ou bargo, et hima ${ }^{3}$. Fango, ou bargo, deux termes d'origine bambara, désignent la force physique, génésique, psychique, sociale,

${ }^{1}$ Cet article est issu d'une enquête effectuée avec Suzanne Lallemand dans le cadre d'une recherche financée par le CNRS et le Ministère des Droits de la Femme (ATP Recherches sur les femmes et Recherches Féministes).

${ }^{2}$ Les Goin constituent une population d'environ 50000 personnes située au sud-ouest du Burkina Faso dans la province de Banfora.

3 La langue goin n'a fait jusqu'à présent l'objet d'aucune transcription officielle. Dans un précédent article nous avions essayé de transcrire les termes vernaculaires dans l'alphabet phonétique international, ce qui eut pour résultat d'en rendre la lecture malaisée. Nous préférons y renoncer cette fois-ci et donner une transcription phonétique françise approximative mais lisible par tous, parti pris renforcé par le fait que la prononciation des mots change d'un village à l'autre, d'un interlocuteur à l'autre et selon le contexte phonétique où il est placé. u se prononce ou, on est nasalisé, mais moins qu'en français. 
économique, administrative, politique. D'un chef, d'une personnalité éclatante, d'un homme fort, d'une terre féconde, de l'autel des ancêtres, on peut dire: u da fango, il a de la force. De celui qui fait des choses extraordinaires, c'est-à-dire impossibles par les moyens ordinaires, tel le prestidigitateur, le guérisseur qui, à la fin de son initiation, retire à main nue des beignets de l'huile bouillante, ou qui plus tard remet sur pied un mourant, on dira: u ke hima, c'est-à-dire il fait des choses extraordinaires. On peut dire aussi : u hi, il fait arriver, sous-entendu des merveilles, des prodiges. Hima désigne donc l'apparition de phénomènes hors de l'ordinaire et, de là, la faculté de susciter ces apparitions.

Un certain nombre d'entités telles que Dieu ou les génies de lieux disposent de hima. Les hommes ordinaires n'en ont pas sauf s'ils l'ont acquis par une initiation : ainsi des guérisseurs (tintama) et des sorciers (kartama). Tama signifie les gens, ceux, et tiyo, celui. Un guérisseur se dit tintiyo, "celui du tin", et les guérisseurs, tintama, "ceux du tin". Le hima des tintama ne peut s'exercer sans des objets-supports, les tinni, sing. tingu. La racine commune à ces mots, tin, se retrouve dans le terme tima, venin, poison 4 . Les guérisseurs sont donc littéralement des empoisonneurs, en ce sens qu'ils empoisonnent ceux qui font le mal, les sorciers, et en ce qu'ils donnent des contre-poisons aux maux physiques et psychiques des individus et de la société. Les tintama agissent au moyen de leurs tinni, rétablissant la santé, le droit, la justice, l'harmonie, en théorie du moins. En réalité, leur initiation leur a conféré la même capacité de hima qu'aux sorciers et I'usage qu'ils en font n'est pas toujours ressenti comme aussi clair et aussi pur que le voudrait la définition de leur rôle.

Pour les puristes de la langue, seuls les objets qui permettent de voir l'invisible méritent le nom de tinni. Cependant, dans la conversation courante, on nomme tinni non seulement les supports

4Nous devons cette étymologie à Marcel Chanal, fin connaisseur de la langue et de la culture goin, qui a bien voulu relire cet article et nous suggérer des modifications. Qu'il trouve ici l'expression de notre reconnaissance. Nous remercions également Alfred Adler et Andras Zempleni pour leurs critiques avisées. 
de pouvoir des tintama mais n'importe quel objet susceptible de se charger de pouvoir par le seul fait qu'on lui a confié un voeu. Si celui-ci concerne la protection de la personne le tingu se nommera kukuyunu, amulette, s'il s'agit d'un souhait maléfique ce sera un kuvangu. Bien qu'ils s'en distinguent par l'identité de leur possesseur, le tingu et le kuvangu sont sémantiquement contigus à une autre espèce de tinni maléfiques : les nidjinani, sing. kudjinango, objets "posés" exclusivement par les sorciers. D'autre part le mot tingu s'emploie couramment pour désigner une réalité nettement différente et qui, stricto sensu se nomme tiufanle. Un tiufanle, ou tiufanlu selon les régions, plur. tiufanga, est un canari rempli d'eau et de racines, affecté à la protection d'un homme et de son groupe familial, que celui-ci soit composé de ses enfants, des membres de sa concession ou de ses maternels. Il est voué à la lutte anti-sorcellerie et veille sur la fécondité, la santé physique et mentale de la famille. Situé à l'intérieur de la concession, il est hérité de père en fils et seul le chef de famille peut accomplir les rites exigés. Il s'apparente donc à un autel familial. Toutefois il arrive qu'un tiufanle de ce genre soit situé à l'extérieur de la concession et que, moyennant paiement, des étrangers puissent lui confier la protection de leurs enfants. Pour les uns c'est alors un tingu, pour les autres un tiufanle, pour d'autres encore les deux à la fois. Certains informateurs estiment que tout ce sur quoi on sacrifie peut se nommer tiufanle; d'autres que le terme tiufanle devrait être réservé aux autels protecteurs de la famille. Les locuteurs accordent donc aux termes tinni et tiufanga un éventail mouvant de significations.

Tout Goin d'un certain âge possède des tinni et des tiufanga. Il est impossible de faire une liste de ces objets car ils ne constituent pas un ensemble fini : chacun peut créer un tingu aujourd'hui et le mettre au rebut demain s'il est inefficace. De nouveaux tinni, originaires des ethnies voisines, sont fréquemment importés en pays goin au gré des voyageurs car les tinni se vendent et s'achètent. De plus les tinni d'origine étrangère, particulièrement ceux des Senufo, jouissent d'une réputation souvent plus prestigieuse que 
les tinni autochtones. Certains tinni goin ont cependant longue vie et sont reconnus par l'ensemble de la communauté goin.

Nous allons passer en revue les principaux tinni et tiufanga en allant des plus stables, des plus largement reconnus, des plus importants symboliquement et socialement, aux tinni mineurs, strictement individuels. A mesure que le consensus à leur égard décrô̂t, leur rôle évolue du pôle positif, celui de protecteur et justicier social, au pôle négatif, celui du sorcier, figure de l'individualisme envieux et destructeur. Cette dégradation de leur prestige et de leurs qualités est parallèle à celle de l'entité donatrice : les tinni les plus forts et les plus sûrs sont donnés par Dieu, ceux qui ne concernent que la protection d'une famille proviennent des ancêtres et des génies de lieux, ceux que l'on peut suspecter des pires intentions sont achetés à un spécialiste ou créés par le désir d'un individu. Par commodité nous répartirons donc les faits selon ces trois chapitres, ce que n'auraient probablement pas fait nos informateurs goin. Pour ne pas trop assécher ni déformer leur pensée, nous présenterons chaque type de tinni dans son contexte à travers les récits des intéressés dont nous avons conservé certaines tournures de style franco-africaines.

Voici, en introduction, une première histoire qui montre les difficultés de nomination des objets de pouvoir et qui illustre l'un de leurs modes possibles d'acquisition :

"Le grand tintiyo L., quatre-vingt-six ans, reçoit encore des clients venus d'Abidjan ou de Ouagadougou. Son pouvoir est cependant faible en comparaison de celui de son père, le redoutable tintiyo $M$. Celui-ci avait pour habitude de tuer les Dyula colporteurs qui passaient par là pour leur prendre leurs biens. L'un d'eux arriva un jour chez M. qui l'accueillit, le traita bien pendant son séjour. Au moment de prendre congé le Dyula dit à son hôte : "Je vais te donner quelque chose pour te remercier. C'est Djumboro. Djumboro est une tiucini pelleon, (litt. vol-queue : une queue de quadrupède pour voler autrui). Que tu pries ou non, ça ne choisit personne, tout le monde peut s'en servir, si tu pries, tu croques de la cola et tu craches dessus, si tu n'es pas musulman, tu la poses sur une daba (la petite houe africaine), tu mets la queue dessus, tu la poses au carrefour, tu prends de la cendre sur le dépotoir, tu fais un cercle autour, tu sacrifies, le sang ne doit pas toucher la queve. Tu lui racontes ton affaire, tu lui fais une promesse; quand ce que tu as demandé se réalise, tu donnes ce que tu as promis, tu verses le sang sur la queue 
à ce moment-là. Je te la donne avec les racines et les secrets nécessaires pour la refaire si elle se perd ou se détruit. Je te donne l'interdit : tu ne dois pas manger de la sauce de feuilles de baobab, ni du pain de singe; de la chèvre, tu peux en manger, mais tu dois te laver avant de sacrifier à Djumboro. Tout ce que tu as dans le coeur, tu le confies à Djumboro, ça se réalise, même si tu as tué quelqu'un, tu t'en sortiras. Tu racontes que quelqu'un a beaucoup d'argent, que tu vas le voler, il faudra que ça marche. Tu vas chercher une tige dans la direction de l'homme, ou de la femme si c'est une femme que tu veux, tu l'attaches à la queue et ça marche" 5 .

Puis le Dyula demanda la route, M. la lui donna et il partit. Quelques heures après, $M$. le rattrapa et le tua pour qu'il ne rappelle pas sa queue. Il lui a tout pris et s'est lavé avec le eneon perro (le "médicament" qui évite les représailles des gens et de certains animaux qu'on a tués ou des mauvais morts qu'on découvre.)".

L'histoire nous fut racontée par un des fils classificatoires de $L$. et confirmée par son frère, tous deux vivant dans la concession de L., en partie initiés à ses tinni à titre d'héritiers potentiels. De leur point de vue, la queue de vol est, parmi l'impressionnant stock de tinni de L., le plus puissant, ce qui ne signifie nullement qu'il est tintiyo grâce à elle. Il est tintiyo parce qu'il fut initié par son père au moyen d'autres tinni.

Un autre informateur, K., doué d'une intelligence exceptionnelle, reprit un jour cette histoire. K. est le neveu utérin de I., il vit dans une autre concession, il héritera des biens matériels de son oncle, mais non de ses pouvoirs magico-religieux. Voici ce qu'il dit :

"Djumboro n'est pas un tingu. On le met dans une peau de macaque et on le range dans sa case, pas dans la case à fétiches; on l'accroche chez soi, on peut lui confier ses problèmes, lui demander des choses, mais ça ne veut pas dire qu'on est initié, qu'on voit les sorciers. Un vrai tingu, on te lave la figure avec et tu vois les sorciers et tu connais les plantes qui guérissent. Djumboro, c'est pas un tingu."

Après quoi, K. qui ne prétend nullement être tintiyo, se remit tranquillement à parler des puissants tinni que lui avait légués son père.

50 note le passage de l'intransitif au transitif selon qu'il s'agit de voler un homme ou une femme. Pour les Goin, voler un homme, c'est lui prendre ses biens, dont sa femme, tandis que voler une femme, c'est la prendre elle-même. 
Donc pour K., lorsqu'il s'agit de son oncle, un tingu désigne exclusivement l'objet de pouvoir qui, après initiation à la confrérie des tintama, permet de voir l'invisible. Simultanément, lorsqu'il s'agit de lui, un tingu désigne tout objet doté de puissance quels que soient sa provenance et ses effets : cette deuxième acception du mot est partagée par I'ensemble des Goin, du moins dans la conversation courante. Venons-en à l'origine des tinni et rappelons qu'ils peuvent être donnés par Dieu, par les génies de lieux, ou institués par soi-même.

\section{I - LES OBJETS DE POUVOIR DONNES PAR DIEU}

Ces objets sont tous en fer, à l'exception des pierres de foudre. Dieu, Dílo, créa d'abord les génies, puis le forgeron et la forge, ensuite le reste de l'humanité; aussi le fer constitue-t-il la médiation la plus directe entre Dieu et les hommes, de même que la forge et l'enclume sont un réceptacle privilégié de la force divine et le forgeron un héros civilisateur. Notons que Dilo désigne Dieu, le ciel et la pluie.

Les objets de pouvoir donnés par Dieu arrivent directement du ciel par le véhicule de la foudre : ce sont d'une part des "pierres de foudre" ou "pierres de pluie" qui auraient été identifiées comme des outils de pierre polie, d'autre part Poìlungo, une lance de fer, et des objets mythiques tels que le premier couteau de l'excision et probablement le dogo, le rhombe de l'initiation. Seul le forgeron peut les recueillir, de même que seul il peut toucher un foudroyé ou éteindre un incendie allumé par la foudre. L'ensemble forgeronforge-enclume-objets de pouvoir en fer fabriqués ou reproduits par le forgeron d'après l'objet initial envoyé par Dieu est pensé comme relevant sans intermédiaire de la sphère divine.

Nous allons nous arrêter successivement sur le dogo, puis sur Poìlungo. 


\section{Le "dogon}

Le dogo, disions-nous est le rhombe de l'initiation: il ne devrait done pas rentrer dans la catégorie des tinni. Cependant, doté de hima, il fonctionne exactement comme un tingu en ce qu'il "attrape" les gens qui transgressent ses interdits, dans le contexte initiatique, mais aussi hors de ce contexte.

Nous n'avons pas trouvé de récit mythique sur l'origine du dogo. Nous savons seulement qu'il fut le premier tingu donné par Dieu à tous les Goin : premier à la fois dans le temps et par son importance symbolique et sociale.

Le dogo est d'abord l'objet symbole autour duquel s'organise l'initiation du même nom. C'est une lame de fer en forme de triangle très allongé que nous appellerons rhombe. Le responsable du dogo le fait tourner et vibrer au-dessus de la tête des néophytes étendus face contre terre la nuit de l'initiation. Il est le "visage de Dieu" qu'ils ne doivent pas voir. Il est gardé soit dans la forge, soit par le chef du groupe d'initiation. Sa puissance est celle de Dieu, relayée par celle de la forge et celle des ancêtres. Le dogo appartient à la communauté goin toute entière, ancêtres, vivants et forgerons. Notons que les voisins, Turka, Karaboro et Toussian, possèdent également un dogo et l'initiation correspondante.

L'initiation consiste à recevoir le nom d'un ancêtre, signe extérieur que l'on est passé d'une continuité biologique avec les ancêtres à une continuité sociale et symbolique. Ce passage n'est pas automatique : les ancêtres appellent qui ils veulent quand ils veulent et nombreux sont ceux qui ne. sont jamais appelés, sans que leur statut social en soit diminué. Un initié puise sa force, sa fécondité, son inspiration, sa chance, sa sagesse, tous termes quion peut traduire par fango, chez les ancêtres, c'est-à-dire du côté du jour, par opposition à la puissance (hima) que I'on peut obtenir des tinni et de la sorcellerie, le côté de la nuit. Le dogo va dans le sens de la collectivité ancestrale contre l'individu, de la bonne connaissance contre la mauvaise, de la paix contre la guerre, de l'harmonie contre la discorde, de toutes les valeurs positives contre les valeurs négatives. De l'initiation on dit qu'elle "arrange les choses de la tête", qu'elle met l'initié 
sur le chemin des ancêtres, qu'elle ouvre la voie vers la seule source de réussite non suspecte : la force (fango) ancestrale. L'initiation est associée à la fécondité spirituelle et génésique. Pour les hommes, une faute contre le dogo est sanctionnée par l'impuissance. En ce qui concerne les femmes, excision et initiation sont liées : l'excision des filles, qui serait leur seule initiation à Soubakaniedougou et la première étape de leur initiation dans le reste du pays, est dite indispensable pour permettre l'accouchement. Dans certains villages, les excisées ne doivent pas pénétrer dans un champ de céréales mûres pendant leur convalescence de peur de dessécher les épis. Le dogo, institution et objet initiatiques, participe ainsi de la constellation Dieu-ciel-pluie-fécondité-forgeforgeron-couteau de l'excision-Poìlungo-ensemble des tinni de fer.

Ce n'est là, sans doute, que l'un des sens de l'initiation. Le signifiant dogo est polysémique, ce qui est particulièrement frappant lorsque les interlocuteurs sont des femmes : invitées à parler de leur dogo, elles peuvent entreprendre le récit de leur excision ou de leur mariage. Si on questionne les intéressées sur les raisons pour lesquelles tant de manifestations sociales peuvent être assimilées ou liées au dogo, elles répondent que le dogo est le chemin des ancêtres, que c'est donc la vie.

Il n'est pas dans notre propos de développer davantage le dogo-initiation mais seulement de montrer en quoi le dogo-tingu, l'objet-dogo, peut sortir du contexte strictement initiatique pour devenir, dans la vie profane, un tingu servant au rétablissement de la concorde, à la protection contre le vol des âmes et des biens matériels.

Symbole d'union et de fécondité, le dogo est utilisé pour apaiser les conflits : autrefois, lors des guerres inter-villageoises, le chef de terre ou l'aîné des forgerons venait se placer au milieu des combattants; il faisait tourner et vibrer la lame de fer au-dessus de leurs têtes et le combat cessait immédiatement. Il sert également à régler les disputes entre groupes ou entre individus : on vo à la forge jurer sur le dogo son innocence ou sa volonté de réconciliation. 
Réceptacle privilégié de forces positives, Dieu, forge et ancêtres, 1 'objet dogo peut être utilisé pour se protéger de tous les maux. Aussi fait-on reproduire par le forgeron des dogo miniatures d'une dizaine de centimètres. Celui-ci les consacre, c'est-à-dire qu'il accomplit le sacrifice et dit l'invocation nécessaire pour leur incorporer la force du dogo, au sens d'entité émanant de la force divine, puis on les suspend partout : dans la concession, pour protéger les habitants contre la sorcellerie, dans les cases, sur les arbres fruitiers, dans les champs, pour les protéger contre le vol. Ces petits dogo sont alors des tinni antivols ordinaires comme il y en a des milliers chez les Goin, en fer, en palme de rônier, en écorce, en terre cuite, en calebasse, en bois, en os, etc. En tant que tingu antivol, son rôle est plus dissuasif que justicier : si un enfant ou un non-initié vole des mangues sur un arbre protégé par un dogo, il ne lui arrivera pas grand mal. On attend du tingu dogo qu'il assure la protection plus que la punition. Il peut protéger les enfants contre les entreprises de sorcellerie mais il ne peut tuer le sorcier qui a réussi à les prendre. Il ne peut être utilisé comme justicier pour le vol d'un bien qu'il n'était pas chargé de défendre.

In revanche il peut être justicier dans sa fonction première: celle de tingu de l'initiation. Si des initiés transgressent ses interdits spécifiques - révèlent par exemple les secrets de l'initiation ou l'utilisent sciemment pour un faux serment - le châtiment, impuissance ou maladie, ne tardera pas.

De l'objet symbole de l'initiation qui soumet les initiés à des interdits spécifiques, ou qui oblige tous les combattants, initiés ou pas, covillageois du propriétaire du dogo et villageois ennemis, à cesser de faire couler le sang, jusqu'aux reproductions miniatures qui protègent des sorciers et du vol, le dogo apparait comme le support et le symbole de toutes les valeurs positives goin : Dieu le leur donna dans des temps primordiaux, l'associa au complexe positif fer-forge-forgeron, en fit une sorte d'arche d'alliance entre eux et leurs ancêtres ; or les ancêtres sont la source de toute vie, de tout ce qui est bon et juste. Aussi le dogo est-il le seul tingu structurellement innocent : il ne peut 
punir que des coupables, il ne peut jamais être utilisé à des fins personnelles qui ne seraient pas dirigées vers la vie et la fécondité.

\section{Poìlungo}

De tous les tinni de fer apportés par la foudre, le plus renommé et le plus puissant est une lance d'environ un mètre soixante-quinze appelée Poǐlungo. On dit que Dieu I'envoya, sans doute au début du siècle, à Yoye Karama, personnage prestigieux, premier d'une lignée de trois chefs de canton illustres pour leur résistance à la colonisation. Poìlungo se trouve à Banfora dans un enclos de ciment adossé au mur d'enceinte de la concession familiale, face à la rue, près de l'une des entrées. L'enclos aurait été construit par un Européen exaucé et reconnaissant. Poìlungo est justicier : il détecte et tue les voleurs ou autres criminels qu'on lui demande de découvrir et de punir. Contre le mur de son enclos, côté rue, chacun peut contempler les témoignages de son efficacité : des dizaines de daba et des monceaux de pagnes sont entassés, appartenant respectivement aux hommes et aux femmes dont il a châtié les méfaits par la mort ; s'y ajoutent les peaux d'animaux et les traces sanglantes des sacrifices offerts durant trois générations.

La victime d'un dommage se rend chez Faduha Hema, descendant agnatique de Yoye Karama et actuel responsable de Poìlungo ; elle lui donne une dizaine de francs et lui expose le motif pour lequel elle veut utiliser la lance ; Faduha remplit un rôle de conseiller et de juge; il fut d'ailleurs membre du tribunal coutumier jusqu'à son abolition par le Président Sankara ; il discute avec le plaignant pour savoir si tous les autres moyens de conciliation ont été employés, si sa cause est juste et si l'utilisation de Poìlungo est légitime. Si Faduha donne son accord, le plaignant emporte chez lui soit Poìlungo lui-même, soit un poìlungo miniature fabriqué par les forgerons et consacré par un sacrifice devant le "tingumère". Il l'apporte dans sa concession, lui indique le délit dont il faut trouver et sanctionner l'auteur, et le rapporte immédiatement chez Faduha. Il ne doit parler avec personne pendant cet aller-retour, 
ou, selon d'autres versions, durant toute cette journée. Lorsque quelqu'un meurt et qu'il est identifié par le devin comme le ou un coupable châtié par Poillungo, le requérant retourne chez Faduha avec les animaux du sacrifice afin de payer les "droits" de Poillungo. Un membre de la famille de Faduha les immole devant le tingu, le remercie et l'avertit que sa mission est accomplie. Faute de cet acte de reconnaissance, Poǐlungo risquerait de continuer aveuglément sa tâche et pourrait "attraper" le requérant ou n'importe qui dans sa concession. Un homme attrapé par Poĭlungo peut sauver sa vie si, l'origine de sa maladie ayant été identifiée à temps par le devin, il avoue et répare ses torts.

Voici un exemple de l'utilisation la plus simple de Poillungo. Le témoignage vient d'un jeune agriculteur du village de Tangora situé à sept kilomètres de Banfora :

"Il y a trois ans (1967) deux boeufs ont disparu à Tangora. Le propriétaire est allé emprunter Poilungo, juste le temps de le mettre dans sa maison et de le prier de faire mourir le voleur. Trois ans plus tard le voleur et son fils sont morts : c'est le devin qui a dit pourquoi. Le propriétaire est alors allé payer $5000 \mathrm{~F}$., deux moutons, dix poulets à Faduha. Les boeufs avaient disparu par sorcellerie; si ça avait été un simple vol, le fétiche aurait agi plus vite. D'autres personnes possèdent des poilungo mais faits par le fonongo (forgeron); celui de Faduha, lui, il vient de la foudre. (...) Il est interdit par la loi d'emprunter un fétiche afin de nuire. (...) Six vaches gardées par les Peul ont disparu. chez Tchombié : il n'est pas allé chercher Poillungo parce que les Peul n'y croyant pas il n'aurait pas d'effet."

Plusieurs choses sont à souligner dans cette déclaration. I'informateur semble subordonner l'efficacité du tingu à la croyance qu'on lui accorde. Si c'était le cas, on serait en droit d'imaginer qu'une telle logique doive limiter la foi des Goin dans leurs tinni. Or il n'en est rien. J. Pouillon a bien montré d'une part la polysémie et l'ambiguĩté du terme croire, d'autre part la relativité culturelle de ce qu'on appelle la "croyance religieuse" (Pouilion 1979 : 43-51). Reprenant ses arguments sur les Hadjerai, nous dirons que la croyance des Goin en Poilungo n'est pas un acte de foi mais la constatation d'un fait d'expérience : on "connâtt" tous les cas où $i 1$ a puni un voleur. Or la même expérience quotidiennne montre que les Peul 
volent sans cesse les boeufs des Goin sans en souffrir. On en déduit que Poillungo n'attrape pas les Peul. C'est pourquoi nous pensons pouvoir affirmer que la remarque de l'informateur est à lire d'abord en sens inverse : Poìlungo n'a pas d'effet sur les Peul, donc ils n'y croient pas. Dans un deuxième temps seulement, on peut se demander pourquoi Poìlungo $n^{\prime}$ a pas d'effet sur les Peul et répondre que c'est parce qu'ils $n^{\prime} y$ croient pas. La pensée est circulaire mais son point de départ est un fait d'expérience : un voleur goin est puni par Poìlungo, un voleur peul non.

L'informateur ressent la nécessité de fournir une excuse à la lenteur de Poĭlungo qui transforme la médiocrité de sa performance en exploit, car tuer un sorcier, un "voleur de nuit", est beaucoup plus difficile que de tuer un simple voleur de jour. Poillungo est donc un vrai tingu au sens strict où l'employait $K$. : un tingu qui voit et tue les sorciers et non un vulgaire petit tingu au sens galvaudé du terme. Puis, ayant reconnu à Poìlungo une puissance (hima) égale mais d'intention opposée à celle des sorciers, l'informateur pose un problème de fond que nous retrouverons tout au long de cet exposé : celui de l'indécise coupure entre pôle bénéfique et pôle maléfique des tinni. Qu'est-ce qui garantit en effet qu'un tingu ne s'éloignera pas de son rôle de justicier et ne tuera que des coupables ? Cette garantie, si elle existe, participe-t-elle de la nature du tingu ou doit-elle être cherchée dans un système de références qui lui est extérieur ? La réflexion du villageois qui fait appel à la législation nationale, ou du moins régionale, montre que la deuxième solution est la bonne. Dans le cas de Poílungo, la loi c'est Faduha et, dans l'opinion des Goin, c'est un citoyen au-dessus de tout soupçon. Mais pour tous les autres tinni et pour tous les autres propriétaires de tinni, nous verrons que la méfiance est permanente. D'autre part, dans un conflit, les torts sont-ils toujours massivement d'un seul côté ? C'était sans doute une vision possible des choses autrefois aux yeux de la coutume, dans le sens le plus juridique du terme. Aujourdhui, dans une ville comme Banfora où se côtoient 35000 personnes représentant toutes les ethnies du pays, le bien ou le mal-fondé des comportements 
peut rarement être tranché par une sanction aussi radicale que la mise à mort de I'un des plaignants.

Voici un exemple de l'intervention de Poilungo vers 1980. On va voir que l'action vengeresse de la lance fut interrompue par l'institution judiciaire.

"A Banfora un homme meurt après être tombé d'un palmier à vin. Un de ses parents lointains vivant à Banfora et le vrai frère du défunt vivant à Abidjan souhaitaient tous deux épouser la veuve. Elle préférait l'homme de Banfora et celui-ci offrit au frère le remboursement de la compensation matrimoniale. Le prétendant d'Abidjan refusa. Ils passèrent outre et se marièrent. Peu de temps après le mari de Banfora tombe gravement malade. Il envoie un émissaire consulter le devin. Celui-ci déclare que la maladie provient de Poîlungo. L'émissaire va interroger Poillungo au moyen d'un sacrifice de poulet : 'Quelqu'un de Banfora a-t-il pris la lance contre le malade ?' Le poulet meurt sur le ventre, signe de réponse négative. Le malade renvoie son ami chez un autre devin : même diagnostic. Il le prie de retourner devant Poillungo et lui dit de poser sa question plus largement. Le messager repart devant Poillungo, sacrifie le poulet et demande si quelqu'un, sans en préciser l'origine, envoie la maladie au mari. Le poulet tombe sur le dos, signe de réponse affirmative de Poİlungo. Le messager s'enquiert alors auprès de Faduha, le propriétaire de Poìlungo, de l'identité de l'agresseur puis, au nom de son ami, il dépose plainte devant la justice contre l'homme d'Abidjan. Les gendarmes convoquent ce dernier et un jugement coutumier est rendu : l'homme d'Abidjan devra accepter le remboursement de $30000 \mathrm{~F}$ CFA de compensation matrimoniale, renoncer à la femme et 'faire sortir' Poìlungo, c'est-à-dire arrêter son action meurtrière. Il lui en coûtera un mouton, plusieurs poulets et $15000 \mathrm{~F}$ à remettre à Faduha. Le mari guérit aussitôt."

Essayons d'apprécier les torts respectifs de chaque partie en regard de la jurisprudence goin, à Banfora, dans les années 80 . Traditionnellement une veuve appartient au lignage maternel de son époux, sauf cas exceptionnels qui sont hors propos. Selon la personnalité du chef de famille, ou bien celui-ci attribue autoritairement la femme à I'un ou l'autre parent, ou bien il autorise des négociations discrètes entre elle et ses prétendants et il respecte le choix de la veuve. Les prétendants évincés se plient généralement à la décision de l'aîné; s'ils la refusent, de longs conflits commencent. La priorité va aux frères utérins du défunt, par ordre d'âge, puis aux neveux directs, puis aux neveux classificatoires, hiérarchie cependant nuancée par le statut conjugal des prétendants, 
les célibataires passant avant les mariés, les monogames avant les polygames. D'autre part, surtout si la veuve n'est plus très jeune et que son nouveau mari demeure loin du premier, elle peut refuser de quitter la concession où elle a vécu jusque-là : certaines familles admettent qu'elle demeure chez son mari défunt et que les nouveaux époux ne se rendent que de rares visites.

Si la veuve refuse de se remarier dans le lignage de son mari, débiteur collectif de sa compensation matrimoniale, et qu'elle fuie avec un amant, trois solutions sont possibles selon le tempérament de "ses maris" :

- ou ils exigent le remboursement de la dot, et son nouveau mariage est légal.

- ou ils la laissent faire sans demander le remboursement, mais sa nouvelle union est illégale et les enfants qui en naitront appartiendront au lignage de son ancien mari.

- ou ils n'acceptent pas de la laisser partir. Dans ce cas ils refusent le remboursement de la compensation matrimoniale et "posent" sur elle un tingu qui a pour mission de tuer sa future descendance, ou son amant ou un membre du lignage de celui-ci. Les "maris" préviennent la femme et l'amant et ils attendent, escomptant la lassitude de ce dernier ou sa peur lors de l'apparition des maladies. Le tingu ne peut fonctionner que si les utilisateurs n'ont pas repris la dot. Cette méthode, la plus sûre pour obtenir le retour de la femme, était autrefois couramment employée dans les villages. Aujourd'hui on contraint de moins en moins les jeunes filles, mais encore assez souvent les veuves, à épouser qui ne leur plâtt pas. La contrainte est évidemment plus fréquente au village et plus rare en ville.

Dans l'affaire qui nous occupe, l'homme de Banfora n'avait pas priorité sur celui d'Abidjan en fonction de leurs liens de parenté respectifs avec le défunt. Mais le premier avait offert le remboursement de la compensation matrimoniale et la femme avait déclaré sa volonté de l'épouser. D'autre part il est difficile d'exiger d'une femre qu'elle quitte sa ville pour aller s'établir à huit cents kilomètres de là. Enfin l'homme d'Abidjan aurait dû prévenir les futurs époux de sa démarche auprès de Poìlungo. Compte tenu. de ces circonstances, Faduha a autorisé, en un premier temps et confor- 
mément à la coutume, I'usage de Poĭlungo pour contraindre l'épouse à rejoindre le frère de son mari ; en un second temps le tribunal coutumier, dont Faduha, a admis la légalité du mariage de Banfora, moyennant le remboursement de la compensation matrimoniale, et a exigé que la vie du nouveau mari soit sauvée.

Un peu surprise néanmoins par la considération que l'administration locale accordait aux manifestations de Poìlungo, nous nous sommes livrée à une enquête auprès des Goin urbanisés et des fonctionnaires de police. Elle révèle que la plupart d'entre eux pensent, qu'avant le Gouvernement Sankara, un homme accusé par Poìlungo était considéré comme un coupable potentiel, qu'on ne pouvait certes condamner sans preuves objectives, mais qu'on pouvait, à titre exceptionnel, "mettre en prison...en attendant...ne serait-ce que pour le protéger de ses accusateurs".

La déclaration du villageois de Tangora selon laquelle il est interdit par la loi d'emprunter un tingu pour nuire est, bien sûr, un aveu d'impuissance à séparer, par un argument décisif et intrinsèque à la logique du système, action bénéfique et action maléfique d'un tingu. Mais dans le contexte de Poìlungo c'est également une déduction logique à partir d'un état de fait réel : si Poǐlungo est utilisé, y compris par la loi, pour punir des coupables, c'est que la loi elle-même garantit qu'il ne peut pas tuer des innocents.

Poìlungo fonctionne mécaniquement, selon un code binaire: il ne peut répondre à une question que par oui ou par non, d'où la nécessité de lui poser la bonne question. On ne peut lui donner qu'un ordre : "tue le coupable". Poillungo est-il faillible et peut-il tuer un innocent? Les Goin veulent croire que non et leurs arguments oscillent entre "Dieu ne le permettrait pas", "c'est interdit par la loi" et "Poìlungo ne se trompe jamais". Poìlungo n'est pas "programmé" pour tenir compte des circonstances atténuantes ou de la relativité d'une faute. Cette responsabilité est dévolue au juge humain, Faduha, qui décide s'il est légitime ou non de le mettre en route. Hormis le dogo, qui n'est pas un justicier, tous les tinni peuvent tuer, que la victime soit coupable ou non. Poìlungo est le seul que l'opinion publique ait placé au-dessus de tout soupçon, non pour une raison structurelle propre à la nature des 
tinni mais pour des raisons contingentes tenant au prestige et à Ia position socio-historique de ses propriétaires. La lignée des chefs possesseurs de Poillungo, la plus illustre du pays goin, incarne les intérêts de cette société pendant la colonisation, pendant les luttes d'indépendance et depuis la constitution de l'Etat voltaique. Personne ne doute d'autre part que Poìlungo ait été envoyé par Dieu et apporté par la foudre à Yoye Karama en personne. Dieu l'a ainsi institué le plus grand tintiyo du pays en même temps qu'il le légitimait comme responsable de région, homme politique et représentant des intérêts goin, double consécration transmise à ses descendants. Il est moins étonnant dens ces conditions que justice divine et justice officielle aient convergé dans les mains de cette famille, noeud symbolique de l'histoire locale.

Poìlungo connât le même processus de reproduction et de miniaturisation que le dogo. Ainsi, pendant leur temps de convalescence, les nouvelles excisées ne lâchent jamais une lance de fer d'un mètre environ nommée également Poìlungo. Elles sont de plus bardées d'amulettes de fer. Certains descendants de fondateur de village ou de quartier, possèdent un poǐlungo hérité de leurs ancêtres qui fonctionne comme tingu protecteur du village. Le villageois de Tangora nous signalait plus haut que "d'autres personnes possèdent des poïlungo, mais faits par les forgerons". Ces lances servent également à la protection contre la sorcellerie et le vol. Ces reproductions sont toutefois beaucoup plus rares que celles du dogo.

II - LES OBJETS DE POUVOIR DONNES PAR LES GENTES DE LIEUX

Rien ne s'oppose à ce que ces objets soient donnés par Dieu mais dans les faits, leur origine est presque toujours attribuée aux génies.

Un mot goin, bimbamba (animaux sauvages, êtres de la brousse) peut être utilisé pour désigner les génies mais personne ne l'emploie jamais. On lui préfère un mot d'origine arabe : djina (plur. dje- 
naba). Les djenaba sont, avant les hommes, les premiers êtres crés par Dieu. Ce ne sont pas des humains mais des personnes vivant en brousse et dont la société fonctionne sur un modèle comparable à celui des Goin : famille, village, etc. Ils sont conformes à la description qu'on trouve dans toute l'Afrique occidentale : petits, blancs, les cheveux lisses et longs, les pieds retournés vers l'arrière, les yeux bougeant sans cesse dans les orbites, la parole hachée, difficilement compréhensible. Ils sont innombrables, habitent la brousse jusqu'à l'orée des concessions qu'ils évitent cependant car ils haïssent le feu domestique, la fumée, les odeurs de cuisine. Ils sont hiérarchisés, sans qu'on sache exactement comment ; ceux. qui vivent dans $I^{\prime}$ eau sont les plus puissants et ils règnent sur un territoire plus large que ceux qui vivent sous ou sur la terre.

Dans l'ensemble les génies sont bien disposés à I'égard des hommes. Certains informateurs considèrent même que Dieu leur a confié la connaissance des végétaux et des animaux de brousse afin qu'ils la transmettent aux hommes. Autrefois ils s'acquittaient de cette mission moyennant des interdits dont le premier était le secret sur l'origine et les circonstances de cette transmission. Aujourd 'hui les hommes, dit-on, boivent trop et ne savent plus garder un secret aussi les génies ne leur confient-ils plus grand chose.

Les dons accordés aux hommes par les génies peuvent se répartir en deux groupes :

A - des connaissances techniques mises au service de la communauté: 1. la divination (siongo : devin).

2. la connaissance des plantes qui guérissent (perontiyo ou tintiyo: guérisseurs).

3. Ia connaissance de l'invisible qui permet de voir et de combattre les sorciers (tintiyo : féticheur en franco-africain). L'origine de cette connaissance peut également être attribuée à Dieu. Elle n'est plus guère transmise directement aux contemporains: elle est héritée ou achetée mais sa possession nécessite de toute façon une initiation à une confrérie de tintama. Il en est de même pour la divination et la connaissance des plantes presque toujours héritées d'un ancêtre lointain et transmises en ligne parallèle : de père 
en fils, et de mère en fille. Le fils ou la fille ne peuvent hériter de cette charge que s'ils ont accompli les rites funéraires pour le parent qui en était responsable avant eux, condition fréquemment transgressée pour les hommes, jamais pour les femmes. Le parent de même sexe peut s'opposer à cette transmission, ce qui arrive rarement. Lorsqu'un guérisseur ou un devin s'apprête à pratiquer son art, l'entité qu'il invoque n'est pas le génie mais son père défunt : il le prie de transmettre offrande et requête à son propre père et ainsi de suite jusqu'à l'ancêtre qui reģut le don du génie, car, seul, ce dernier connất l'identité de ce génie et les modalités précises de l'alliance. Aussi peut-on dire que la connaissance de ces techniques n'est pas due au seul génie mais à la conjonction des forces bienveillantes du génie et du père (ou de la mère), conjonction dans laquelle la place du père est de loin la plus importante.

B - La seconde catégorie de dons faits aux hommes par les génies se distingue de la précédente en ce qu'elle ne concerne que le destinataire et sa descendance directe. Sont de ce type :

1. un trésor inépuisable de cauris.

2. divers supports de pouvoir plus ou moins spécialisés : pour protéger contre la sorcellerie ou la maladie, pour avoir beaucoup d'enfants, pour aider au succès des chasseurs, pour protéger les troupeaux ou les récoltes, pour "attacher" les forces des rivaux dans les compétitions de culture, pour empêcher qu'un autre attache vos propres forces, pour assurer des succès économiques, amoureux, sportifs, scolaires, guerriers, bref pour être le plus fort dans toutes les circonstances de la vie. Nous ne parlerons ici que de cette dernière catégorie de dons.

Parmi ces supports de pouvoir il faut à nouveau distinguer deux types :

a) Ceux qui sont destinés à la lutte anti-sorcellerie. Ils protègent la santé physique et mentale ainsi que la fécondité d'un homme et de son groupe familial. Ces objets de protection ne sont pas des tinni mais des tiufanga; on ne les nomme tinni que si, dépassant la sphère strictement familiale, ils peuvent être utilisés, moyennant 
paiement, par des étrangers à la famille ou à la concession. Leur vocation est exclusivement protective, ils ne peuvent agresser ou punir. Leur forme matérielle est toujours la même : un canari rempli d'eau du marigot, demeure du génie qui fit ce don à l'ancêtre, et des éléments végétaux, généralement des racines, provenant de ce même marigot ou de son voisinage immédiat. Le canari s'accompagne souvent d'une queue de cheval ou de quadrupède, instrument privilégié dans l'ensemble du pays goin pour chasser les sorciers. Ce choix viendrait d'une extension de sa fonction première de chasse-mouches 6 . Ces tiufanga sont toujours référés à des génies de lieux, mais comme ils sont généralement hérités du père, exceptionnellement des maternels, l'entité invoquée est le père défunt; le génie n'est pas nommé. Le propriétaire se contente d'une formule telle que: "Mon père, c'est toi qui m'as légué ce tiufanle, voici la poule que je t'offre, bois et transmets aux ancêtres. Garde les enfants en bonne santé." Le médiateur paternel s'est peu à peu substitué au donateur originel de sorte que les rites accomplis pour ces tiufanga doublent le culte ancestral pratiqué sur des autels spécifiques : ils n'en sont qu'une modalité particulière. La différence serait, pour les uns, qu'on s'adresse à l'autel ancestral sur un signe des ancêtres et aux canaris sur sa propre initiative, et pour les autres, l'inverse.

b) Une seconde catégorie d'objets supports de pouvoir donnés par les génies concerne tous ceux qui sont préposés, non plus à la protection des principes vitaux de la personne, mais à aider celle-ci dans les circonstances accidentelles de la vie, que ce soit en augmentant ses forces, en les protégeant d'éventuelles agressions extérieures ou en "attachant" les forces de ses ennemis ou de ses concurrents. Ces tinni peuvent prendre toutes les formes et être constitués de n'importe quelle matière minérale, végétale ou animale. Toutefois les génies ne sont que l'un des pourvoyeurs possibles de ces objets dont l'origine n'est pas toujours claire.

Voyons maintenant les différentes modalités possibles de la rencontre et de l'alliance entre le génie et l'homme.

${ }^{6}$ Comnunication personnelle de Marcel Chanal. 


\section{Les modalités de la rencontre}

Le don d'un objet de pouvoir peut être le résultat d'une rencontre personnelle entre un homme et un génie qui veut lui témoigner de l'amitié. Le génie est dissimulé sous la forme d'un animal sauvage quelconque, mais sa présence ou son comportement considérés comme inhabituels avertissent qu'il s'agit d'un génie. Voici une histoiretype de ce genre de rencontre faite le plus souvent par les chasseurs:

"Un homme va à la chasse. Il guette des cobas 7 et s'apergoit qu'un lion les guette aussi. Les cobas arrivent, l'homme ne bouge pas. Le lion tue deux cobas et s'en va sans eux. I'homme attend, atterd, puis comprend que le lion lui a fait un cadeau. Il rentre chez lui avec les cobas. Plus tard il tombe malade, consulte, le devin lui dit qu'il n'a pas remercié le lion, qu'il doit partir à l'endroit où il l'a rencontré et sacrifier un poussin, puis se coucher par terre : il verra dans l'arbre une calebasse qu'il décrochera. Elle contient un puissant tingu pour la chasse."

Parfois l'homme capte par la violence la puissance du génie. En voici un exemple :

"Un chasseur voit en brousse, posé par terre, un petit canari rempli d'une décoction de plantes. Il veut le soulever pour se laver mais il ne parvient pas à le décoller du sol. Alors il attrape le liquide du bout des doigts et s'en frotte le corps. Au bout d'un moment il a assez de force pour incliner le canari. Quand il s'est lavé entièrement, il monte sur un arbre et attend. Un animal fabuleux arrive car il a senti que son canari a été dérangé. L'homme descend, affronte I'animal. Il a maintenant la même force que l'animal et l'intelligence en plus : il le terrasse. I'animal lui demande la vie sauve en échange de ses secrets. I'homme accepte, écoute ses secrets puis le tue. C'est obligatoire sinon l'animal reprend ses secrets et on devient tout vide.

La rencontre entre le génie et l'homme est généralement immatérielle. L'homme rêve sans voir ni entendre précisément un génie et il va chez le devin faire interpréter son rêve obscur. Il apprend alors qu'il doit aller sacrifier à tel endroit de la brousse car un génie veut lui faire un don. Le signe de cet appel peut également être une maladie. Certains résistent à l'appel des génies, tel ce Goin cuisinier à Abidjan qui n'avait aucune envie d'être devin,

\footnotetext{
7 Coba ou antilope cheval (Hippotragus equinus).
} 
dont le bras avait enflé et ne désenfla que lorsque le malade fut revenu au village pour installer une case de divination. Le tingu ou le tiufanle peut être le résultat d'une rencontre non plus amicale et voulue par le génie, mais provoquée involontairement par l'homme et suscitant la colère du génie :

"Par exemple, un homme enfreint l'interdit d'un djina de passer à tel endroit; il tombe malade; le devin lui dit d'aller faire un sacrifice là-bas, de prendre de la terre et une branche de l'endroit, de les rapporter à la maison; ça devient la force du tiufanle : on apprivoise."

La rencontre est souvent due à I'initiative humaine. Un homme exprime une demande à un arbre, une colline, un marigot, que ces lieux soient anonymes ou déjà réputés pour la puissance de leur génie ; il sacrifie et promet d'autres sacrifices s'il est exaucé. Puis un échange régulier s'instaure entre les deux partenaires, l'homme demandant au génie pouvoir ou protection et lui offrant des sacrifices, soit de sa propre initiative, soit lorsque le génie réclame à travers le processus maladie-rêve-interprétation du devin. Très souvent le génie offre à l'homme d'étendre sa protection sur ses enfants ou sur les membres de sa concession. L'homme rapporte alors à la maison des fragments végétaux, de la terre ou de I'eau de l'habitat du génie. Selon les indications du devin, il les met dans un canari et lave toute la famille avec la décoction: le tiufanle est constitué. Chaque concession possède ainsi des séries de tiufanga hérités du père ou acquis par soi-même. Les femmes n'ont pas le droit de constituer un canari ni de toucher à cette eau : elles ne peuvent que demander à un homme, père, frère ou mari, d'aller sacrifier pour elles à l'endroit où elles ont été en contact avec le génie, ou dans cette direction.

\section{Du marigot à la concession}

D'où vient le pouvoir contenu dans le tiufanle ? Pourquoi les tiufanga sont-ils presque toujours constitués d'eau et d'éléments végétaux provenant du lieu de rencontre entre l'homme et le génie? Quelle relation unit le contenu du canari et l'homme, le génie et les constituants de la personne ? 
Le pouvoir du tiufanle est dit provenir principalement des racines baignant dans le canari, ou du moins être déposé en elles. Sur l'origine de la puissance des végétaux, il n'y a pas d'ambiguïté: aucun végétal n'a de hima par lui-même. Le hima qui agit à travers lui est celui du génie, utilisable par les hommes après manipulation rituelle. Le hima n'est lié à aucune espèce végétale particulière et le fait qu'il soit déposé dans l'un de ses représentants ne signifie pas qu'il existe dans tous : il est présent dans les seuls exemplaires croissant dans l'aire de pouvoir du génie et ayant subi la manipulation rituelle requise. Toutefois ceci ne semble s'appliquer qu'aux tiufanga familiaux car les tintama professionnels utilisent préférentiellement certaines espèces particulières. La manipulation rituelle consiste en une séquence de gestes, d'invocations, de dispositions spatiales, diinterdits accompagnant la cueillette, le traitement et l'utilisation des plantes. Si on se trompe dans cette séquence rituelle, on ne peut pas mobiliser la puissance du génie, "exactement, m'a dit un informateur, comme ton magnétophone: si tu n'appuies pas sur le bon bouton, ga ne marche pas." Lorsqu'on a obtenu de quelqu'un un tingu et son secret, l'autre ne l'a pas pour autant perdu. Si on l'a obtenu par la force, l'autre vous le reprendra immanquablement et se vengera. D'où le meurtre du Dyula par le tintiyo après qu'il en ait reçu le secret de la queue-de-vol, et celui du génie-animal fabuleux après que le chasseur lui eût extorqué ses secrets. Pour ces raisons, les tinni s'héritent ou s'achètent. Il est impossible d'utiliser un tingu contre son vendeur car le lien entre celui-ci et le tingu demeure après la transmission.

Le statut du canari du génie-animal fabuleux n'est pas clair: est-ce un tingu que chacun peut utiliser même sans en connâtre le secret; ou est-ce un tiufanle de protection contenant une partie des principes vitaux du génie? Dans l'un ou l'autre cas, comment l'utilisation mécanique de la décoction peut-elle vider le génie de ses forces au profit du chasseur ? Quelle relation unit le génie à son canari ? Nous passerons en revue un peu plus loin les différentes hypothèses envisageables.

Un des aspects de la relation marigot-contenu du canari est certainement d'ordre métonymique. Pour les Goin, les génies sont 
strictement localisés et les éléments prélevés dans leur aire d'habitation contiennent leur pouvoir. Le culte rendu au canari ne dispense pas son propriétaire d'aller sacrifier de temps en temps au lieu d'origine de ce pouvoir. Il semble que la relation entre le lieu d'origine et l'élément prélevé soit pensée sur le modèle suivant.

Dans le sud-ouest du pays goin, un culte villageois a lieu normalement tous les deux ans ou en cas de danger. Il se nomme nya nelle "chasser le village", expression dont personne ne paraissait connâtre le sens jusqu'à ce que le gardien des tinni d'un village nous propose l'explication suivante : dans la concession du fondateur de ce village, on garde des queues d'animaux et le couteau du sacrifice, qui constituent les tinni protecteurs du village. Leurs propriétaires, les nellentama ("village-ceux"), c'est-à-dire les descendants agnatiques du fondateur, accomplissent régulièrement dans la concession les sacrifices nécessaires pour garantir l'harmonie entre les villageois, éviter les malheurs, demander la santé, la pluie et de bonnes récoltes. Mais tous les deux ans, ou en cas de danger, les nellentama, après avoir accompli ces sacrifices dans la concession, partent pour un lieu sacré du village situé en brousse sacrifier un chien et un coq rayé de rouge. S'ils laissaient passer trop de temps sans faire ce sacrifice les génies quitteraient les tinni de la concession et repartiraient en brousse vers leur lieu d'origine, aussi les vieux s'y rendent et chassent (nya) les génies vers le village (nelle). On dit aussi: ba ka bi ba ba dio, "ils vont les appeler pour qu'ils viennent". Le nellentiyo, le responsable, dit par exemple aux génies : "Autrefois on avait telle ou telle chose, maintenant on ne l'a plus, donc je vous donne ce poulet pour que vous vous leviez et arrangiez ça". Puis il sacrifie et les nellentama mangent sur place la viande des animaux sacrifiés. Personne ne doit voir la cérémonie et personne ne sort en brousse ce jour-là. Après le rituel, les génies les suivent au village jusque dans leur concession. Le but de l'opération est donc de recapter et de réapprivoiser cette puissance sauvage au profit des hommes, de rétablir le passage entre deux mondes opposés : la brousse et le village.

Cependant un autre interlocuteur conteste l'origine du tingu de la lignée des fondateurs. Il dit que le sacrifice accompli sur 
la queue, à l'intérieur de la concession, ne s'adresse pas à un génie mais à l'ancêtre premier occupant du lieu. Cette queue n'aurait rien à voir avec un génie localisé : en effet les queues données par les génies résultent d'une apparition tandis que celle-ci a été fabriquée par les ancêtres du premier occupant sur les indications du devin. La base de la queue est effectivement prise dans une boule ovoìde composée de racines pilées et les indications du devin concernent l'espèce végétale et les manipulations rituelles requises. Une fois le tingu achevé, l'ancêtre l'aurait consacré en sacrifiant un poulet à Dieu en disant : "Quand j'ai un problème, de même que les habitants de ce village, je te le confie pour que tu le règles." C'est pourquoi il s'en sert pour "arranger" les affaires du village.

Ces deux versions de l'origine du tingu villageois nous semblent conciliables. Nous avons vu en effet que lorsque l'origine d'un tingu était très ancienne, ses utilisateurs ne s'adressaient plus à l'entité donatrice mais exclusivement à la lignée ancestrale transmettrice. Peu importe finalement que Dieu ait donné le tingu aux ancêtres par l'intermédiaire du devin ou du génie; en revanche demeurent pertinents les matériaux dont il est fait, en l'occurrence racines et queues d'animal sauvage, car ils attestent que sa puissance appartient au domaine de la brousse. D'autre part, si le tingu du fondateur du village est tellurique, il n'est pas autochtone puisque, par définition, celui-ci est venu d'ailleurs. Il nous semble donc logique que les descendants du fondateur, d'une part sacrifient dans leur concession pour demander à leurs ancêtres de maintenir leur force vivante dans le tingu, d'autre part aillent périodiquement sacrifier en brousse, là où ils avaient autrefois demandé accueil et bénédiction au génie du lieu, le priant de renouveler cette alliance. Le bien-être des villageois dépend de Dieu, des ancêtres du fondateur du village et du génie du lieu qui seul peut transformer les tinni étrangers en tinni autochtones. M. Izard a bien montré que l'extériorité et l'errance étaient des attributs essentiels aux fondateurs de village et que l'association génie-étranger définissait l'autochtonie (Izard : 1979, 1983).

Toutefois la niétonynie n'épuise pas tous les aspects de la relation marigot-canari. 
On constate que presque tous les Goin rendent un culte à un ou plusieurs marigots. Ce lieu de culte leur a été légué par leur père ou par leurs maternels, ou ils l'ont inauguré eux-mêmes, ou encore ils sacrifient là où leur diufwengo sacrifiait. Le diufwengo est la composante spirituelle essentielle de la personne : c'est la part de l'ancêtre réincarnée dans un nouvel être, le double de chacun au monde des ancêtres, le gardien de yale, l'âme. C'est le diufwengo qui livre l'âme au sorcier ou au contraire lui en interdit l'accès. I'identité ancestrale du diufwengo est secrète et seuls, en principe, l'intéressé et ses géniteurs la connaissent. Pour les Goin, une personne se compose de trois parties : diufwengo, yalé et kwoma, le corps. Yale se manifeste par trois phénomènes perceptibles aux sens humains : yale, la voix; yale, l'ombre; yale, le reflet (miroir, photo...). On peut de manière métonymique atteinảre un homme par tous les objets avec lesquels son corps et les trois manifestations de yale ont été en contact, ou par le nom de son diufwengo dans la mesure où celui-ci révèle son identité humaine précédente, mais on ne peut pas parler de ces différents éléments comme des constituants de la personne : ce sont des traces.

Il arrive que le diufwengo réclame un sacrifice : comme toutes les autres entités, il avertit son double humain au moyen d'une maladie. Le devin consulté informe alors le malade qu'il doit aller sacrifier soit sur l'autel où le diufwengo figure comme ancêtre, soit au marigot où il sacrifiait de son vivant. Le malade s'y rend et adresse le sacrifice à son diufwengo comme si le marigot était sa demeure. De même, Iorsqu'un homme, sur son initiative ou sur l'orare du devin, sacrifie à son père, il peut aller soit devant l'autel ancestral, soit au marigot où son père sacrifiait de son vivant. Si on demande à l'intéressé pourquoi il honore son père défunt au bord d'un marigot plutôt que sur l'autel destiné à cet usage, il répondra que son père faisait ainsi avant lui et que cette coutume date d'une alliance offerte par le génie du lieu à un de leurs ancêtres, alliance dont les termes exacts sont tombés dans l'oubli. Mais on a parfois l'impression que cette réponse est une rationalisation a posteriori et que le lien entre l'entité invoquée, père ou diufwengo, et le génie du lieu n'est pas de l'ordre 
de l'échange cultuel - un pouvoir ou une protection contre un sacrifice - mais d'un échange beaucoup plus intime, comme d'une communauté de nature ou d'origine.

Ainsi, certaines formules couramment employées dans le langage quotidien laisseraient croire que chacun possède un double, un jumeau, un principe directeur ou protecteur au marigot. Par exemple, de deux personnes qui s'aiment, on dit que les génies de leur tête s'entendent bien; d'une femme volage que son génie est jaloux et ne lui permet pas de s'attacher durablement à un homme.

D'autre part les femmes vont très fréquemment implorer le marigot de leur donner des enfants. Or les Goin, interrogés sur le rôle des génies dans la conception, maintiennent obstinément qu'une naissance normale ne doit rien aux génies. Il arrive qu'un génie amoureux d'une femme entre dans son ventre : mais l'enfantgénie mourra généralement très vite. A un nouveau-né malade ou trop faible on administre un "médicament" (tingu) afin de connaître sa nature : si c'est un génie, il meurt, si c'est un bébé humain, il survit à l'épreuve.

Une autre théorie, avancée par quelques-uns, démentie par d'autres, affirme que certains diufwelma empêchés de nâtre plusieurs fois par sorcellerie - dans le cas d'une femme qui a fait des fausses couches répétées - et dégoûtés de la méchanceté humaine, se détourneraient quelque temps de leurs descendants et se réfugieraient au marigot. Lorsque le désir leur en revient, ils pénètrent dans l'utérus d'une femme venue prier au bord de l'eau, évitant ainsi le passage par le village. Mais ces retraites aquatiques des doubles ancestraux seraient rares.

Et pourtant, cette mystérieuse liaison entre génies de l'eau et diufwengo peut se manifester également à l'autre extrémité de la vie. Voici le récit de la mort du tintiyo M. par son fils, le tintiyo $\mathrm{L}$.

"Mon père savait le jour et I'heure de sa mort. Il me l'avait dit lontemps avant. La veille il a dit à ses enfants d'aller au marigot tuer un oiseau inattrapable. Ils y sont allés et l'ont tué facilement. Puis le soir il leur a dit : 'J'ai vu les ancêtres sacrifier pour moi un coq et une pintade'. Le lendemain matin il leur a dit : 'Avez-vous renversé le canari 
qui est là dehors ? - Non - Vous voulez donc que le soleil se lève ancore une fois ?' Ils ont renversé le canari et quand ils se sont retournés vers leur père, celui-ci était mort."

Les tintama racontent souvent que leur père (et non pas euxmêmes car les pouvoirs se dégradent et le père défunt est toujours plus puissant que son fils) avait à son service des oiseaux, toujours posés près de lui, dont seul il pouvait voir la nature de génie. L'oiseau semble donc être l'objet de deux représentations distinctes: d'une part génie protecteur d'une personne, d'autre part double de l'homme ou du moins support d'une partie de ses principes vitaux. La même dualité s'applique à son canari : en tant que tiufanle il contient des éléments venus du marigot, donc du génie ; mais, dans la mesure où le fait de le renverser fait mourir son propriétaire, il contient quelque chose de ses principes vitaux. Nous ne connaissons, il est vrai, aucune histoire du même type pour un homme ordinaire et on peut aussi envisager que cette capacité de dédoublement soit propre aux grands tintama arrivés à un haut degré d'initiation et de savoir.

Cependant si l'on compare la fin du tintiyo $M$. et celle du génie-animal fabuleux, on voit que les deux récits ont la même structure. Les génies eux-mêmes, dont les Goin ne savent pas s'ils ont un diufwengo ou non, semblent avoir leur double dans l'eau d'un marigot. Malgré les dénégations des intéressés, on ne peut plus guère douter que chaque être possède, en la personne d'un génie de l'eau, une sorte de jumeau. D'ailleurs, lorsqu'un enfant naît génie, les Goin imaginent que des négociations ont eu lieu entre l'ancêtre qui souhaite recommencer son cycle de vie humaine dans le ventre de la future mère et le génie qui prend sa place : les uns disent que le génie a peut-être tué un foetus humain déjà en place et renvoyé le diufwengo au monde des ancêtres, d'autres pensent qu'il n'y a jamais épreuve de force mais toujours entente amiable entre génies et ancêtres.

Si la participation respective des génies et des ancêtres dans le processus de la conception ne fait l'objet d'aucun consensus dans les représentations goin, leur collaboration est attestée, sans précision mais avec certitude, dans d'autres domaines. Ainsi 
le devin, généralement une femme, est le personnage central de la vie goin. On se souvient que la divination est un don des génies. Pour devenir devin, une femme doit avoir accompli le rite funéraire, le ambalma dont nous parlerons plus loin, pour son ascendante, mère et grand-mère classificatoires, devins avant elle, car "les génies n'abandonnent jamais la descendance de celui ou celle avec qui ils ont fait alliance." Deuxièmement elle doit avoir été initiée au dogo, "avoir été mise sur le chemin des ancêtres", sinon les génies ne lui parleront pas.

Mais s'agit-il des génies de l'eau ou des génies de lieux ? Sont-ils de nature identique ou différente. Ce sont les mêmes, répondent tous les Goin, à l'exception d'une vieille informatrice qui les distingue. Pour elle, les djenabe se trouvent dans les grands arbres tandis que les génies de l'eau ne sont pas des djenaba mais des humma noma (eau-personnes). Lorsque les hummanoma célèbrent leurs mariages et leurs funérailles, on peut entendre leur balafon. Ils se transforment en humains et se promènent parmi nous, surtout pendant les mariages et les funérailles. Ils boivent le dolo (bière de mil) qu'on leur offre mais refusent de manger du poisson. Si on touche leur corps, il est frais et gélatineux comme celui du poisson électrique. En revanche les djenaba ne viennent jamais aux fêtes des hommes.

Que penser de cette opinion dans un monde où le consensus sur les représentations collectives est faible ? Est-ce la réminiscence d'une vision du monde commune et oubliée ou une création personnelle ? Nous l'ignorons. Cependant, compte tenu d'une part, de l'étroite liaison attestée par les faits entre génies de l'eau et naissances humaines, d'autre part de son absence entre celles-ci et génies de la brousse, cette distinction parât logique.

Pour l'instant, nous ne sommes pas en mesure d'éclairer davantage les représentations goin sur le rôle des génies dans la conception des humains ni sur les relations entre génies et ancêtres, génies et diufwengo. Nous savons que les génies participent au processus de la conception chez certains peuples de l'aire voltaíque 
(Gourmantché, Mossi...) 8 . Il est possible que les Goin aient partagé autrefois ce fond de croyances, mais actuellement ils ne disposent pas de représentations d'ensemble sur ces questions : seuls demeurent les morceaux d'un puzzle dont le sens global se serait perdu.

Il nous semble cependant raisonnable de penser que si le contenu d'un tiufanle a pouvoir sur la vie d'une personne, si en même temps ce contenu est support de la puissance d'un génie de l'eau, c'est qu'un lien étroit doit exister entre génie de l'eau et constituants de la personne. Le lieu de cette conjonction se situe dans l'eau du marigot et dans les eaux amniotiques, sans que l'on sache si la corrélation entre ces deux milieux aquatiques est ou était plus qu'une simple métaphore.

\section{III - LES TINNI CONSTITUES PAR LE DESIR DיUN HOMME}

Il n'est pas nécessaire de rencontrer un génie pour acquérir un tingu. N'importe qui peut en constituer un avec n'importe quel objet naturel ou fabriqué. Il suffit de lui confier son désir et le yale, l'âme de l'invocateur imprègne l'objet. Des trois manifestations sensibles de yale, la voix, l'ombre et le reflet, la voix est le véhicule privilégié de cette imprégnation. Un autre mot, nelma, signifie paroles, message, intentions exprimées. Or les informateurs précisent bien que ce qui constitue la puissance du tingu n'est pas le contenu du message (nelma) mais son instrument sonore, yale, la voix, terme qui peut également désigner les supports modernes d'un message telles qu'une lettre ou une bande magnétique. Ils veulent sans doute exprimer non pas que le message est indiffé-

${ }^{8}$ Sur les Gourmantché voir: CARTRY M., "Du village à la brousse ou le retour de la question. A propos des Gourmantché du Gobnangou (Haute-Volta)" in La fonction symbolique. Essais d'anthropologie réunis par M. Izard et P. Smith, Paris, Gallimard, NRF, 1979, 265-288. Sur les Mossi voir : BONNET, D. Corps biologique, corps social. Les Mossi de Haute-Volta. Thèse de troisième cycle, Paris, EHESS, 1982. 
rent, ce qui serait un non-sens, mais que la force qui capte et contraint une puissance à exécuter le voeu exprimé est yale, la voix, déposée dans l'objet. Autrement dit, c'est la voix qui est "fétichisée".

Une malédiction - yale (+nelma) - lancée en l'absence de support matériel peut attirer des ennuis à son destinataire mais n'atteindra jamais l'efficacité du tingu - support matériel + yale (+nelma) sauf dans quelques cas exceptionnels : la malédiction du père ou de la mère contre ses enfants, celle proférée contre un vrai frère lorsque le nom de Dieu a été invoqué. Dans ces deux cas un sacrifice doit être accompli par le forgeron pour arrêter l'effet maléfique et permettre la réconciliation.

D'où vient donc la puissance d'une voix lorsqu'elle a confié son désir à un arbre, un caillou, une colline, un morceau de fer ou n'importe quoi ? L'invocation semble créer cette puissance à partir de rien : celle-ci n'est référée à aucune origine, elle s'engendre d'elle-même; la réussite du souhait et la punition qui frappe l'invocateur s'il omet de remercier le tingu par le sacrifice promis prouvent son existence. Souhait et promesse sont également dépourvus de destinataire précis. Le tingu nous semble alors être parfaitement caractérisé par ces lignes de Jean Pouillon : "... le fétiche est ce qu'on ne parvient pas vraiment à penser. (...), il est une limite. L'écart, que tout lien symbolique suppose pour le surmonter peut, ou bien se creuser, ou bien se réduire. Se creuser au point de presque rompre le lien et rien, si ce n'est un mot rendu quasiment vide, n'arrive à rendre l'immatérialité du sens : le mot abstrait est signe, n'est que signe. Se réduire au point de rendre presque indiscernable la différence entre la signification et le support matériel qui lui est associé : le fétiche est signification, n'est que signification. Le signe verbal et le fétiche remplissent ainsi de fonctions analogues, puisque grâce au mot on prétend fixer l'idée et, selon une formule expressive, le saisir : les mots sont des pièges à idées comme les fétiches des "pièges à dieux". Mais ils s'opposent, puisque, si les mots n'étaient que des fétiches, la sémantique se ramènerait à la phonologie." (Pouillon $1970: 146)$. 
Cependant un "piège à dieux" sans destinataire - pour les Goin un "piège à hima" - est ressenti comme dangereux car il pourrait capter un mauvais hima. Aussi s'efforce-t-on de se prémunir contre ce risque en invoquant le nom de Dieu par une formule de ce genre: "Dieu, j'ai fait ce tingu en ton nom, je l'arrose en ton nom, fais que j'obtienne telle ou telle chose. "Puis on sacrifie un poulet pour savoir si Dieu accepte de donner sa caution. Le voeu exaucé, on va consulter le devin pour savoir ce qu'on doit et à qui. On peut aussi se référer à un génie mais la garantie divine est plus prestigieuse. Tant que le souhait est positif on peut s'en remettre à I'omniprésence de Dieu et des génies. Mais si le voeu que l'on confie à un caillou, par exemple, est maléfique, à qui s'adresse-t-on ? Peut-on penser que Dieu ou les génies accepteront un tel souhait ? Et si ce voeu maléfique a été exaucé, quelle puissance l'a pris en charge?

Les manipulations licites et illicites des puissances surnaturelles de même que le glissement possible des unes aux autres constituent une préoccupation majeure des Goin. Voici deux exemples de ce glissement, le premier vu par un témoin extérieur, une voisine, le second par un témoin "intérieur", le devin.

Un paysan, par ailleurs très honorablement connu et responsable dans son village de l'office Régional de Développement, avait un interdit de parole, plus des interdits alimentaires et sexuels deux jours par semaine, soit deux jours sur cinq (durée de la semaine goin). Il m'expliqua qu'il avait été longtemps champion de culture, que pendant un temps sa daba était devenue miraculeuse et qu'il ne pouvait m'en dire plus. Il me montra une daba miniature pendue à son cou.

Quinze ans plus tard, en ville, c'est-à-dire dans un lieu éloigné spatialement et culturellement du village où ils vivaient tous deux, une de ses voisines me raconta l'histoire suivante:

"Le père de ce monsieur avait fait planter du mil par une petite fille vierge et le mil germa en une nuit ; il en fit de la farine, il y mit un tingu et le donna à son fils pour que les gens ne puissent pas l'"attacher" pendant les compétitions de culture. Mais le monsieur a "travaillé" le tingu pour attacher les autres. C'est pour ça que les enfants de ses neveux sont morts." 
Dans l'esprit de la voisine, la première opération, de défense, relève du tintiyo ou d'un tingu et elle est licite. La preuve : personne n'est mort. La deuxième, l'agression, bascule dans la sorcellerie. La preuve: la mort des enfants de neveux, qui sont des fils classificatoires.

Je n'ai jamais pu savoir ce que cet homme payait exactement par son lourd interdit mais, vraisemblablement, il s'agissait soit de l'interdit lié à un pouvoir dont l'origine était attribuée à un génie ou à Dieu, mais encore une fois il paraft anormalement pesant, soit du prix de sa culpabilité pour avoir été trop longtemps le meilleur cultivateur de son village, suprématie consacrée de surcrôt par les services administratifs.

Le thème de la daba surnaturelle est très fréquent chez ces passionnés d'agriculture. Il constitue une séquence qui peut se lire dans les deux sens : un homme se livre volontairement à des manipulations magiques licites (tingu) ou illicites (pacte de sorcellerie) afin d'obtenir une daba extraordinairement compétitive; mais il peut également arriver qu'une daba devienne tout à coup extraordinairement compétitive sans que son propriétaire ait entrepris aucune démarche magique, comme si une puissance inconnue s'était mise spontanément à son service. Le champion de culture, talonné par les soupçons d'autrui, se sentira en danger tant qu'il ne saura pas ce qu'il doit payer et à qui. La déclaration de cet ancien chef de canton sorti de I'Ecole Nationale d'Administration illustre le second cas :

"Il arrive que votre daba devienne surnaturelle, vous êtes toujours premier dans les compétitions, vos champs donnent plus que ceux des autres. Alors il faut absolument que vous alliez sacrifier et que vous portiez sur vous une daba miniature."

Comme je demandais à qui on devait sacrifier, il me répondit qu'il fallait interroger le devin.

Ainsi, tout succès peut devenir source de culpabilité pour son auteur et de suspicion pour son entourage, car tout succès a tendance à être interprété en termes d'aide surnaturelle et toute infortune en termes de transgression des conditions exigées pour cette aide. Les moyens de capture de cette aide surnaturelle et leur 
légitimité inspirent nombre de métaphores dans le langage courant, voire des procédés rhétoriques destinés à les mettre à distance. Ainsi, deux de mes informateurs, parents agnatiques vivant dans la même concession, m'avaient consacré beaucoup de temps. Je leur témoignai ma reconnaissance par un cadeau envoyé de France. A mon retour l'aîné me remercia en ces termes : "Les gens vont croire que nous t'avons mise dans notre queue...", simple image, me rassura mon interprète, pour exprimer des relations si chaleureuses qu'on pourrait croire qu'elles furent obtenues au moyen du tingu en forme de queve de quadrupède.

Voici le deuxième exemple de glissement d'un usage licite à un usage illicite des forces surnaturelles. Particulièrement riche, il nous servira de conclusion car il met en scène la quasi totalité des tinni dont nous avons parlé. L'histoire est racontée par le même interprète, directeur d'école primaire qui, en tant que fils classificatoire des protagonistes et intellectuel de la famille, intervient dans la plupart des épisodes. Ceux-ci se déroulent en trois temps - 1976, 1984, 1986 - et, bien que le lien entre le premier et les deux suivants n'apparaisse pas immédiatement, il est évident qu'ils forment un tout dans l'esprit du narrateur.

Voici le schéma familial des acteurs; les noms sont des pseudonymes et les numéros renvoient à l'ordre des décès :

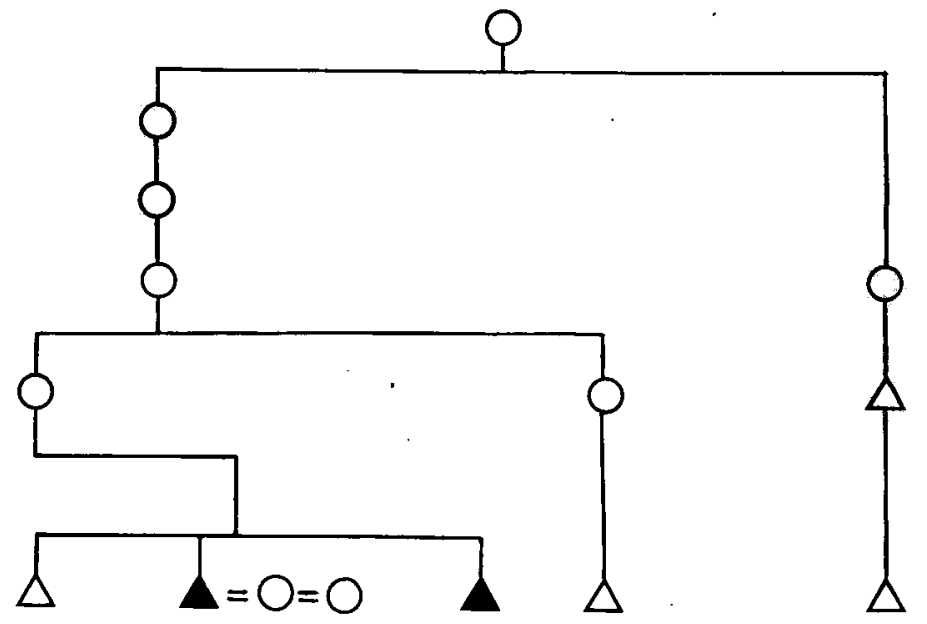

Kamon

Tol1o

Mambia

Tchikan

L'informateur 
Premier épisode, vers 1976 :

"Il y a une dizaine d'années Mambia meurt en Côte-d'Ivoire et laisse une assurance-vie de 400000 F CFA environ. A Soubakaniedougou son frère Tollo cache la somme dans un canari de la concession. $200000 \mathrm{~F}$ disparaissent. Il consulte des devins, des tintama, des marabouts et dépense beaucoup d'argent sans résultat. I'un d'eux lui demande $5000 \mathrm{~F}$ et il les enterre dans une case en sa présence. Tollo devait les déterrer au bout d'une semaine et les retrouver avec les $200000 \mathrm{~F}$ disparus. Aidé de l'informateur il rouvre le trou et constate seulement la disparition des $5000 \mathrm{~F}$. Il va chercher Poìlungo. Il perd deux enfants, son frère Tchikan en perd un."

Huit ans s'écoulent pendant lesquels il ne se passe rien : ni l'argent ni le voleur ne sont retrouvés et il ne sera plus question de ces événements dans la suite du récit. Le deuxième épisode débute vers 1984 :

"Il y a environ deux ans Kamon perd de l'argent. Il va chercher Kanoro, un tingu: il ressemble à un balai de pailles ou de branches de néré. On passe le cou de quelqu'un dans les pailles: s'il est coupable, elles serrent. Trois personnes sont ainsi 'étranglées' : Tollo, un vieux et une vieille de la concession. Pour la vieille qui ne sait pas compter, c'est plus que douteux. Tollo s'est senti humilié par Kamon. Il va chercher en cachette le igale (un caillou) du ambalma (rituel de deuil), mais n'importe quel caillou aurait pu faire l'affaire, il suffit de parler dessus. Il a dit : 'Puisque mon grand-frère m'a fait cette honte, que mes biens sont ceux de mon frère, si jamais je meurs que le grand-frère ne prenne rien, ni biens, ni gens, sinon que le igale le prenne'. Donc le igale était devenu un tingu, les paroles suffisent, il n'y a pas besoin de sacrifice."

Deux années s'écoulent à nouveau sans incident. Le troisième épisode intervient en février 86 :

"Tollo meurt brusquement. On consulte le devin. Celui-ci révèle l'existence d'un tingu (le igale), et dit qu'il faut sacrifier. On fait venir le fonongo (le forgeron), le poulet tombe mal. Tchikan dit alors à son grand-frère Kamon : 'Dans ce cas, moi non plus je ne peux rien prendre de l'héritage. Donc les femmes vont rester comme ça (c'est-à-dire sans mari). Il faut que nous retournions consulter chacun de notre côté.' Kamon y va et revient avec la même histoire de igale mais, d'après le devin, le sacrificateur aurait dû être un nyubingo (quelqu'un portant le même nom de matriclan) et pas un étranger puisque la querelle est familiale. Tchikan, lui, revient avec une autre affaire : 'Tollo una tu isso Dilo', litt. Tollo avait pris nuit Dieu (il avait fait de la sorcellerie avec Dieu lui-même). Done là, il faut un sacrifice du fonongo (forgeron). De plus il avait posé un kudjinango, un objet maléfique, sur 
chacune de ses deux femmes. Il les avait obtenus d'un kartiyo (sorcier) : pour les enlever il faut un tintiyo (anti-sorcier) qui sacrifie un poulet. On parle dessus, ca suffit, après le sorcier le lavera pour le faire devenir sorcier, pour qu'il voie l'invisible, puis il l'enseignera. Donc les deux frères ont recommencé les deux sacrifices et les deux poulets sont bien tombés. Kamon et Tchikan vont pouvoir prendre chacun une femme : elles sont d'accord."

Reprenons la succession de tinni, de spécialistes et d'entités à l'oeuvre dans cette affaire depuis dix ans :

1 - Devins, tintama, marabouts (certains manifestement véreux) chargés de trouver et de punir les voleurs, plus les innombrables devins consultés à chaque étape du processus. La confiance dans les devins n'étant pas totale, lorsqu'il s'agit d'une affaire familiale, on envoie plusieurs membres de la famille en consultation et autant de fois qu'il est nécessaire pour obtenir des avis concordants.

2 - Poìlungo. Est-ce lui qui a tué les trois enfants ? Ce n'est pas dit et ce ne peut pas l'être car les enfants étaient trop petits pour avoir dérobé l'argent. Cependant la juxtaposition des deux propositions : "Il va chercher Poìlungo" - "II perd deux enfants, son frère Tchikan en perd un" révèle le rapprochement qui s'est opéré dans l'esprit de l'informateur et le doute qui y est demeuré. On ne saura jamais qui a pris cet argent, mais on ne saura pas non plus la cause exacte de la mort de Tollo, dont voici les circonstances: cet homme de quarante ans, en pleine santé apparente, s'est levé vers le milieu du jour pour aller se reposer sous son arbre à quelques mètres de la concession, et il a demandé à sa femme de lui apporter de l'eau. Quand elle est arrivée il était mort. C'est une mauvaise mort car il n'a pas rendu l'âme sur sa natte dans sa case mais dehors sur la terre. Le rituel de son enterrement a été écourté et il n'aura pas de funérailles complètes. Aux yeux de tous, une telle mort est la conséquence d'une faute ou d'une transgression. L'interlocuteur ne dit pas laquelle et le devin ne l'a peut-être pas dit non plus. Cette imprécision est en effet indispensable à la crédibilité de Poîlungo et des autres tinni, la croyance qu'on leur accorde s'alimentant moins de la preuve de leur efficacité 
que de l'impossibilité de prouver leur inefficacité ou leurs erreurs. Cependant la vérité devrait en principe être connue lors des funérailles de Tollo qui auront lieu l'année prochaine.

3 - Kanoro : c'est un de ces multiples petits tinni dont on ignore l'origine exacte et dont la voix publique porte la réputation plus ou moins loin et plus ou moins longtemps. Son responsable demeure à Diagora, un village voisin; lui-même ou un délégué se déplace à la demande, il place le tingu autour du cou des suspects en disant : "Kanoro, si c'est lui qui a volé, attrape-le." Pourquoi Kanoro a-t-il attrapé une "vieille qui ne sait pas compter" et un vieux de la concession dont il ne sera plus question ainsi que Tollo? Pour l'instant l'identité du voleur et les causes de la mort de Tollo gardent suffisamment de mystère pour que Kanoro ne soit pas acculé aux preuves de son imposture ou de son obsolescence.

4 - Le igale du ambalma : n'en déplaise à mon interprète, ce n'est pas n'importe quel caillou mais un eaillou qu'on utilise comme tingu à defaut de, ou en plus des tinni spécialisés de la concession, lors du rituel le plus important et le plus périlleux de la vie sociale goin : le ambalma. Le ambalma est le rituel funéraire au cours duquel, d'une part on transforme un cadavre en ancêtre, d'autre part on sépare du mort ses veuves et ses enfants. Ceux-ci courent, durant ce passage, un danger mortel de par les résistances du défunt et à cause des assauts en sorcellerie qui convergent sur eux. On prétend que chacun ne peut faire le ambalma qu'une seule fois dans sa vie car personne n'est assez fort pour le subir deux fois sans mourir (mais ce n'est pas la seule raison pour laquelle on ne le fait qu'une fois.) Aussi les proches adjurent-ils Dieu, à travers les tinni, de renvoyer sur leur tête leurs éventuels désir de meurtre. A Soubakaniedougou par exemple, l'un des bidama du mort (autrefois ses compagnons de guerre, aujourd'hui les descendants agnatiques des compagnons de guerre de ses pères) prend un caillou, igale, le pose sur sa tête et dit : "Nous, tes bidama, sommes venus faire ton ambalma. Celui qui veut faire du mal aux ambalmatama (les veuves et les enfants), que Dieu ne le laisse pas faire. Nous faisons la coutume de nos vieux. Celui qui va se lever pour faire du mal, que Dieu le tue". Puis il pose le caillou sur la tête de chacun des 
participants en répétant ces paroles. Le igale du ambalma est donc déjà constitué en un redoutable tingu.

5 - Le forgeron : son intervention est obligatoire chaque fois qu'une malédiction emprunte sa puissance directement à Dieu. Nous avons vu plus haut que le forgeron appartenait lui aussi sans intermédiaire à la sphère divine. Cette symétrie de position du forgeron et de l'invocateur par rapport à la divinité permet au premier, et à lui seul, d'interrompre le redoutable processus engagé par le second.

6 - Le nyubingo (nyu : mère) : lorsque quelqu'un a maudit un membre de sa famille, il doit appeler un nyubingo, c'est-à-dire n'importe quel homme portant le même nom de clan, afin que celui-ci arrête par un sacrifice les effets de sa parole meurtrière.

7 - Le kartiyo, le sorcier : il a été sollicité par Tollo pour doubler l'action du igale et de la sorcellerie avec Dieu car les nidjinani (plur. de kudjinango) ont pour but de tuer ses frères si, comme la coutume le requiert, ils épousaient ses veuves. Mais pourquoi Tollo envisage-t-il la possibilité d'une mort imminente? D'abord on ne peut obtenir l'aide d'un sorcier sans qu'il vous initie à la confrérie. Le hima du sorcier une fois obtenu, il faut payer en donnant des âmes, celles de ses enfants ou la sienne. Quant à Dieu, on ignore le prix qu'il demande lorsqu'on lui fait jouer le rôle de sorcier suprême. Enfin il reste l'énigme non résolue de l'identité des deux voleurs et les menaces que Poìlungo et Kanoro font peser sur eux.

8 - Le tintiyo : c'est l'inévitable partenaire du sorcier puisqu'il a seul vocation pour désamorcer son travail.

On remarquera que le igale du ambalma est un tingu tandis que les nidjinani sont des objets de sorcellerie. Or ils remplissent exactement la même fonction, seul leur mode d'acquisition diffère.

Essayons de préciser davantage ce qui distingue et ce qui lie les trois objets : tingu donné par une entité, tingu créé par un homme et objet support de sorcellerie. Il n'existe pas, à notre connaissance, de sorcellerie innée en pays goin, mais seulement 
une sorcellerie acquise par contrat et initiation auprès d'un sorcier. Les tingu achetés à un tintiyo réputé, ou garantis par une partie de l'opinion publique, tel Kanoro, ou par Dieu ou la totalité de l'opinion publique, tel Poìlungo, sont des pièges à hima dont on pense qu'ils ont, à des degrés divers, fait leurs preuves. On pense aussi qu'ils ne fonctionneront pas "pour faire le mal", pour tuer un innocent. Pour Poìlungo on en est (presque) sûr, pour les autres on l'espère. L'axiome selon lequel un tingu publiquement reconnu ne peut faire le mal, quelle qu'en soit par ailleurs la raison, a pour conséquence de rejeter tout tingu acquis personnellement du côté des intentions de nuire, du côté de la sorcellerie. Cependant il existe une différence essentielle entre le tingu du type igale et un objet de sorcellerie comme kudjinango : c'est la question du prix. Du monde sorcier, les Goin ne savent rien (il faut être sorcier pour savoir, disent-ils), hormis une chose : son prix exorbitant, appréhendé comme contre-valeur exacte du pouvoir qu'il confère. I'utilisation du igale revient alors à une tentative de sorcellerie où on s'instituerait soi-même sorcier pour n'avoir pas à en payer le prix : ce déni des lois de l'échange est précisément ce qui voue l'entreprise à l'incertitude.

Les vieux disent que les génies ne font plus de dons aux hommes et qu'eux-mêmes ne transmettent plus la totalité de leurs connaissances magiques aux jeunes car ceux-ci n'ont plus la force de respecter les interdits très durs qui en étaient le prix, qu'en conséquence ils risqueraient a'en mésuser. La communication du hima aux hommes était subordonnée à une ascèse qui d'une part, était nécessaire à la non-dégradation de cette énergie supra-humaine, d'autre part témoignait de la qualité et de la force du destinataire et garantissait sa capacité de n'en pas faire mauvais usage. Au-delà du discours banal sur la disparition des splendeurs d'antan, les vieux disent aussi quelque chose de bien connu sur le prix de l'accès au symbolique. Rompant avec l'unanimité de ce point de vue sur la décadence des jeunes, un informateur inversa les termes du problème et dit : "Les jeunes ne veulent plus des secrets des vieux parce qu'ils ont peur que ga les rende sorciers." C'est bien toujours du prix qu'il 
est question. Cette opinion, à première vue contradictoire avec les précédentes, les conforte en réalité : les jeunes ignorent l'ascèse qui était le juste prix parce que les conditions économiques ont changé. Ils vont (parfois) à l'école et migrent six mois par an en Côte-d'Ivoire pour gagner de l'argent. Ecole et salariat sont les seuls moyens licites qu'ils connaissent pour acquérir savoir et pouvoir, car le coût en est, sinon prévisible, du moins visible, puisqu'il est extérieur au domaine occulte. Les frontières du licite et de l'illicite, les efforts et les dangers qu'ils impliquent, sont, on le voit, une constante de la pensée goin, ce qui n'a rien d'étonnant dans une société, d'une part obsédée par la sorcellerie, d'autre part en rapide mutation.

La création par un homme d'un tingu maléfique est donc une tentative pour obtenir sans payer un hima aussi puissant que celui "vendu" par le sorcier. Pour les Goin eux-mêmes c'est un leurre. D'où 1 'accumulation des procédés maléfiques attribuée à Tollo par les devins : elle révèle le peu de crédit qu'il accordait à ses initiatives et leur incapacité à compenser l'humiliation dont elles s'originaient. D'où aussi le flottement permanent des sens du mot tinni conformément au modèle : "Je sais bien que ce ne sont pas de vrais tinni, mais quand même..." (Mannoni, 1969).

Parallèlement ces maléfices accèdent, au-delà du problème de leur efficacité, à un absolu d'existence grâce à l'oracle du poulet. A partir du moment où Tollo meurt, on met en route un appareil rituel complexe pour lui permettre de devenir ancêtre. Nul ne peut devenir ancêtre s'il est entaché du crime de sorcellerie ou d'utilisation de tinni meurtriers. Le monde des ancêtres est a-sorcier. De longs et minutieux interrogatoires du mort suivis de sacrifices de purification se succèdent jusqu'à ce que, par le poulet, le défunt atteste qu'il est lavé de ses souillures et que les ancêtres Iui ont ouvert la porte. Or l'oracle par le sacrifice des poulets est un moyen de communication avec l'au-delà jamais mis en doute. Tant que Tollo était vivant personne ne pouvait savoir s'il avait utilisé des maléfices, lui-même ne pouvait pas savoir si ceux-ci avaient réussi à piéger un hima quelconque puisque l'héritage de 
ses veuves, seul instrument de preuve, ne se serait jamais posé. Le passage par la mort est le seul moment de vérité, celui où le défunt, contraint par les ancêtres, avoue s'il était sorcier et ce qu'il a fait. Il revient au monde des ancêtres - parce qu'il exclut la sorcellerie - de la dénoncer et, par là, d'en administrer la preuve au monde des vivants. La parole d'un mort est infaillible, à plus forte raison une fois qu'il est devenu ancêtre. Autrefois, lorsque le mort avait confessé la liste de ses crimes, les familles des victimes prenaient dans son lignage autant d'enfants qu'il avait reconnu avoir tués.

Cependant tant que le sorcier est vivant, les ancêtres peuvent éventuellement protéger ses futures victimes mais ils n'interviennent pas contre lui : seuls les tintama le font et ils sont trop proches du mal qu'ils combattent pour être totalement fiables. Dans le dernier récit, l'informateur donne une description spontanée de l'apprentissage du sorcier : "...le sorcier le lavera pour le faire devenir sorcier, pour qu'il voit l'invisible, puis il l'enseignera." Qu'on compare cette déclaration avec celle de $\mathrm{K}$. décrivant l'apprentissage du tintiyo et on verra que la distance entre les deux est mince : "Un vrai tingu, on te lave la figure avec et tu vois les sorciers et tu connais les plantes qui guérissent." Les Goin ont d'ailleurs du mal à imaginer les relations tintama-sorciers; pour certains si un tintiyo devenait sorcier, il mourrait ou ses tinni perdraient leur pouvoir. Mais la plupart imaginent des négociations, souvent mercantiles, entre eux, témoin cette réflexion: "Je ne vois pas pourquoi les tintama se plaignent des sorciers puisqu'ils en vivent." Conscients du fait que les deux personnages sont l'avers et le revers du même phénomène, les Goin ont souvent tenté de se débarrasser de ce couple maudit en se portant en masse vers les prophètes à tendance monothéiste qui se lèvent régulièrement dans la région. Ainsi, vers les années 60, sous I'influence de Moussa de Sinématiali (Côte-d'Ivoire), ils jetèrent tous leurs tinni et tiufanga pour être délivrés de la sorcellerie. Mais ils les reprirent au bout de deux ou trois ans car "dans le malheur on ne peut pas être seul avec Dieu." 
Les tintama et leurs tinni poursuivent, de manière fort ambiguë, un but, I'éradication de la sorcellerie, que chacun juge inaccessible ici-bas car, finalement, seules la mort et l'ancestralité permettent d'y parvenir.

Michèle Dacher E.H.E.S.S.

\section{Ouvrages de référence}

IZARD M.

1979 Transgression, transversalité, errance in La fonctịon symbolique. Essais d'anthropologie réunis par M. Izard et P. Smith, Paris, Gallimard, NRF, 289-306.

1983 Engrammes du pouvoir : l'autochtonie et l'ancestralité, Le temps de la réflexion, 4, 299-323.

MANNONI 0.

1969 Clefs pour l'imaginaire, Paris, Seuil.

LE MOAL G.

1980 Les bobo ; nature et fonction des masques, Travaux et documents de 1'ORSTOM $n^{\circ} 121$, ORSTOM, Paris, 535p.

POUILLON J.

1970 Fétiches sans fétichismes, Nouvelle Revue de Psychanalyse, $2,135-147$.

1979 Remarques sur le verbe "croire" in la fonction symbolique..., Paris, Gallimard, NRF', 289-30. 